\title{
Lower bounds for the football pool problem for 7 and 8 matches
}

\author{
Wolfgang Haas \\ Albert-Ludwigs-Universität \\ Mathematisches Institut \\ Eckerstr. 1 \\ 79104 Freiburg, Germany \\ wolfgang_haas@gmx . net
}

Submitted: Oct 26, 2006; Accepted: Mar 15, 2007; Published: Mar 28, 2007

Mathematics Subject Classifications: 94B65

\begin{abstract}
Let $k_{3}(n)$ denote the minimal cardinality of a ternary code of length $n$ and covering radius one. In this paper we show $k_{3}(7) \geq 156$ and $k_{3}(8) \geq 402$ improving on the best previously known bounds $k_{3}(7) \geq 153$ and $k_{3}(8) \geq 398$. The proofs are founded on a recent technique of the author for dealing with systems of linear inequalities satisfied by the number of elements of a covering code, that lie in $k$ dimensional subspaces of $\mathbf{F}_{3}^{n}$.
\end{abstract}

\section{Introduction}

Let $\mathbf{F}_{3}=\{0,1,2\}$ denote the finite field with three elements. The Hamming distance $d(\lambda, \mu)$ between $\lambda=\left(x_{1}, \ldots, x_{n}\right) \in \mathbf{F}_{3}^{n}$ and $\mu=\left(y_{1}, \ldots, y_{n}\right) \in \mathbf{F}_{3}^{n}$ is defined by

$$
d(\lambda, \mu)=\left|\left\{i \in\{1, \ldots, n\}: x_{i} \neq y_{i}\right\}\right| .
$$

The subset $C \subset \mathbf{F}_{3}^{n}$ is called a ternary code with covering radius (at most) one, if

$$
\forall \lambda \in \mathbf{F}_{3}^{n} \exists \mu \in C \quad \text { with } \quad d(\lambda, \mu) \leq 1
$$

holds. For a monograph on covering codes see [1]. The problem to determine $k_{3}(n)$, the minimal cardinality of a ternary code with covering radius one is known as the "football pool problem" and was widely studied during the last decades. Updated bounds for $k_{3}(n)$ are contained in an internet table by Kéri [7].

The easy bound

$$
k_{3}(n) \geq \frac{3^{n}}{2 n+1}
$$


is known as the sphere covering bound. In the recent papers [2], [3] the author developed a new technique (based on a method of Habsieger [4]) to improve on the sphere covering bound by dealing with systems of linear inequalities satisfied by the number of elements of $C$, that lie in $k$-dimensional subspaces of $\mathbf{F}_{3}^{n}$. The method presented in [3] is limited by $k \approx \frac{n}{2}$. The reason is, that for larger values of $k$ the "irregular" solutions of the linear inequalities no longer yield a negligible amount in the necessary estimations.

The aim of this paper is to present a first method to deal with these irregularities. We consider the cases $n=7$ and $n=8$ (with $k=4$ resp. $k=5$ ). In the case $n=7$ the best previously known lower bound $k_{3}(7) \geq 153$ is due to Habsieger [5]. We show

Theorem 1. $k_{3}(7) \geq 156$.

For the case of 8 matches the best lower bound $k_{3}(8) \geq 398$ is due to Habsieger, Plagne [6] and the author [3]. Here we show

Theorem 2. $k_{3}(8) \geq 402$.

In section 2 we state the system of linear inequalities mentioned above (Lemma 1) and limit the degree of irregularity of its solutions (Lemma 2). In section 3 we start with some preliminaries. Section 4 contains a proof of $k_{3}(7) \geq 155$. The more detailed considerations needed to prove Theorem 1 are contained in section 5 . In section 6 we prove Theorem 2 .

We remark, that the proofs in this paper do not use any computer calculations.

\section{The covering inequalities}

The definitions in this section are used in the whole paper. We say that $\mu \in \mathbf{F}_{3}^{n} 1$-covers $\lambda \in \mathbf{F}_{3}^{n}$ if $d(\lambda, \mu) \leq 1$. For $\sigma \in \mathbf{F}_{3}^{k}$ and $1 \leq j \leq k(\leq n)$ we define one-dimensional subspaces of $\mathbf{F}_{3}^{k}$ (lines) by

$$
\begin{gathered}
L(\sigma, j)=\left\{\rho \in \mathbf{F}_{3}^{k}: \rho \text { and } \sigma \text { differ at most in the } j \text { th coordinate }\right\}, \\
\mathcal{L}=\left\{L(\sigma, j): \sigma \in \mathbf{F}_{3}^{k}, 1 \leq j \leq k\right\} .
\end{gathered}
$$

It is clear, that $|L|=3$ holds for all $L \in \mathcal{L}$. Apparently $L\left(\sigma_{2}, j_{2}\right)=L\left(\sigma_{1}, j_{1}\right)$ is equivalent to $j_{2}=j_{1}$ and $\sigma_{2} \in L\left(\sigma_{1}, j_{1}\right)$. This implies

$$
|\{L \in \mathcal{L}: \sigma \in L\}|=k \quad \text { for each } \quad \sigma \in \mathbf{F}_{3}^{k}
$$

and

$$
|\mathcal{L}|=k 3^{k-1} .
$$

If $x_{\sigma}$ is a nonnegative integer for each $\sigma \in \mathbf{F}_{3}^{k}$, we say that the triple $\left(x_{\sigma}, x_{\rho}, x_{\tau}\right)$ is the value distribution of the line $L=\{\sigma, \rho, \tau\} \in \mathcal{L}$. We write

$$
g(L)=\sum_{\sigma \in L} x_{\sigma} \quad \text { for each } \quad L \in \mathcal{L},
$$




$$
\mathcal{L}_{i}=\left\{L \in \mathcal{L}: \max _{\sigma \in L} x_{\sigma}=i\right\}, \quad \mathcal{L}_{\geq i}=\bigcup_{j \geq i} \mathcal{L}_{j} \quad \text { for } i \geq 0 .
$$

For $\lambda \in \mathbf{F}_{3}^{n}$ we define $h_{k}(\lambda) \in \mathbf{F}_{3}^{k}(1 \leq k \leq n)$ by

$$
h_{k}(\lambda)=\left(x_{1}, \ldots, x_{k}\right) \quad \text { if } \quad \lambda=\left(x_{1}, \ldots, x_{k}, \ldots, x_{n}\right) .
$$

Now assume $C \subset \mathbf{F}_{3}^{n}$ is a ternary code. For $\sigma \in \mathbf{F}_{3}^{k}(1 \leq k \leq n)$ we set

$$
A_{\sigma}=\left\{\lambda \in \mathbf{F}_{3}^{n}: h_{k}(\lambda)=\sigma\right\}, \quad n_{\sigma}=\left|C \cap A_{\sigma}\right| .
$$

Finally we set

$$
k_{i}=\left|\left\{\sigma \in \mathbf{F}_{3}^{k}: n_{\sigma}=i\right\}\right| \quad \text { for } \quad i \geq 0 .
$$

Lemma 1A (Habsieger [4]) If $C \subset \mathbf{F}_{3}^{n}$ has covering radius one and $k=n-3$ we have

$$
7 n_{\sigma}+\sum_{\rho \in \mathbf{F}_{3}^{k}, d(\rho, \sigma)=1} n_{\rho} \geq 27 \text { for } \sigma \in \mathbf{F}_{3}^{k}
$$

Proof. By (1) the 27 elements of $A_{\sigma}$ have to be 1-covered by $C$. This can be done only by the elements of $C \cap A_{\sigma}$ and $C \cap A_{\rho}$ with $\rho \in \mathbf{F}_{3}^{k}, d(\rho, \sigma)=1$. Since each element of $C \cap A_{\sigma}$ 1-covers 7 elements of $A_{\sigma}$ and each element of $C \cap A_{\rho}$ one, (6) follows.

Lemma 1B If $C \subset \mathbf{F}_{3}^{n}$ has covering radius one and $k=n-3$ we have

$$
\sum_{L \in \mathcal{L}: \sigma \in L} g(L) \geq 27-(7-k) n_{\sigma} \quad \text { for } \quad \sigma \in \mathbf{F}_{3}^{k}
$$

concerning the numbers $n_{\sigma}$ defined in (5).

Proof. By (2) we have

$$
\sum_{L \in \mathcal{L}: \sigma \in L} g(L)=k n_{\sigma}+\sum_{\rho \in \mathbf{F}_{3}^{k}, d(\rho, \sigma)=1} n_{\rho}=7 n_{\sigma}+\sum_{\rho \in \mathbf{F}_{3}^{k}, d(\rho, \sigma)=1} n_{\rho}-(7-k) n_{\sigma}
$$

for $\sigma \in \mathbf{F}_{3}^{k}$ and Lemma 1B follows from Lemma 1A.

We now show, that large values of $n_{\sigma}$ cannot occur "too often".

Lemma 2 Assume $C \subset \mathbf{F}_{3}^{n}$ is a ternary code with covering radius one and $k=$ $n-3$. Moreover assume that, after a suitable permutation of the coordinates of the code, whenever $\rho \in \mathbf{F}_{3}^{k}$ with $n_{\rho} \geq 5$, then the set $C \cap A_{\rho}$ 1-covers at most s from the 27 elements of $A_{\rho}$. Then

$$
k_{4}+\sum_{i \geq 5}(7 i-s) k_{i} \leq 6|C|-3\left\lfloor\frac{3^{n}-|C|}{n}\right\rfloor-\min \left\{3,3^{n}-|C|-n\left\lfloor\frac{3^{n}-|C|}{n}\right\rfloor\right\} .
$$


Proof. For $1 \leq i \leq n$ we set

$$
\left.C_{i}=\left\{\lambda \in \mathbf{F}_{3}^{n} \backslash C: \exists \mu \in C \text { ( } \lambda \text { and } \mu \text { differ exactly in the } i \text { th coordinate }\right)\right\} .
$$

By (1) we have $\mathbf{F}_{3}^{n} \backslash C=\bigcup_{1<i<n} C_{i}$. We choose pairwise disjoint sets $C_{i}^{\prime}(1 \leq i \leq n)$ satisfying $C_{i}^{\prime} \subset C_{i}(1 \leq i \leq n)$ and $\bigcup_{1 \leq i \leq n} C_{i}^{\prime}=\bigcup_{1 \leq i \leq n} C_{i}$. After a suitable permutation of the coordinates of the code we may assume $\left|C_{1}^{\prime}\right| \leq\left|C_{2}^{\prime}\right| \leq \ldots \leq\left|C_{n}^{\prime}\right|$. Write $3^{n}-|C|=q n+r$ with integers $q, r$ and $0 \leq r<n$. We find

$$
3 q+\min \{3, r\} \leq\left|C_{n-2} \cup C_{n-1} \cup C_{n}\right| .
$$

Assume to the contrary $\left|C_{n-2} \cup C_{n-1} \cup C_{n}\right|<3 q+\min \{3, r\}$. Then $\left|C_{n-2}^{\prime}\right|+\left|C_{n-1}^{\prime}\right|+\left|C_{n}^{\prime}\right| \leq$ $\left|C_{n-2} \cup C_{n-1} \cup C_{n}\right|<3 q+3$ implying $\left|C_{n-2}^{\prime}\right| \leq\left\lfloor\left(\left|C_{n-2}^{\prime}\right|+\left|C_{n-1}^{\prime}\right|+\left|C_{n}^{\prime}\right|\right) / 3\right\rfloor \leq q$ and thus $q n+r=\left|\mathbf{F}_{3}^{n} \backslash C\right|=\left|\bigcup_{1<i<n} C_{i}\right|=\left|C_{1}^{\prime}\right|+\ldots+\left|C_{n}^{\prime}\right| \leq(n-3)\left|C_{n-3}^{\prime}\right|+\left|C_{n-2} \cup C_{n-1} \cup C_{n}\right|<$ $(n-3) q+3 q+\min \{3, r\} \leq q n+r$, a contradiction.

Now for $\sigma \in \mathbf{F}_{3}^{k}$ we set

$$
S(\sigma)=\left\{\lambda \in A_{\sigma} \backslash C: \lambda \text { is } 1 \text {-covered by } C \cap A_{\sigma}\right\} .
$$

We then have $C_{n-2} \cup C_{n-1} \cup C_{n}=\bigcup_{\sigma \in \mathbf{F}_{3}^{k}} S(\sigma)$. Since $\mu \in C \cap A_{\sigma}$ 1-covers at most 6 elements from $A_{\sigma} \backslash C$ and $\left|A_{\sigma}\right|=27$ we have $|S(\sigma)| \leq \min \left\{6 n_{\sigma}, 27-n_{\sigma}\right\}$. Moreover, if $n_{\sigma} \geq 5$, by the proposition of the Lemma we may use the estimation $|S(\sigma)| \leq s-n_{\sigma}$. We now find

$$
\begin{aligned}
\left|C_{n-2} \cup C_{n-1} \cup C_{n}\right| & \leq \sum_{\sigma \in \mathbf{F}_{3}^{k}}|S(\sigma)|=\sum_{1 \leq i \leq 3} \sum_{\substack{\sigma \in \mathbf{F}_{3}^{k} \\
n_{\sigma}=i}}|S(\sigma)|+\sum_{\substack{\sigma \in \mathbf{F}_{3}^{k} \\
n_{\sigma}=4}}|S(\sigma)|+\sum_{i \geq 5} \sum_{\substack{\sigma \in \mathbf{F}_{3}^{k} \\
n_{\sigma}=i}}|S(\sigma)| \\
& \leq 6 \sum_{1 \leq i \leq 3} i k_{i}+23 k_{4}+\sum_{i \geq 5}(s-i) k_{i} \\
& =6 \sum_{i \geq 1} i k_{i}-k_{4}-\sum_{i \geq 5}(7 i-s) k_{i}=6|C|-k_{4}-\sum_{i \geq 5}(7 i-s) k_{i}
\end{aligned}
$$

and Lemma 2 follows by (7), $q=\left\lfloor\frac{3^{n}-|C|}{n}\right\rfloor$ and $r=3^{n}-|C|-q n$.

Lemma 3 If $C \subset \mathbf{F}_{3}^{7}$ is a ternary code with covering radius one, $|C| \leq 155$ and $k=4$, then without loss of generality we may assume

$$
k_{5}+2 k_{6}+3 \sum_{i \geq 7} k_{i} \leq 7 .
$$

Proof. After a suitable permutation of the coordinates of the code (which does not affect the covering radius) we may apply Lemma 2 with $n=7$ and $s=27$ and get

$$
8 k_{5}+15 k_{6}+22 k_{7}+29 \sum_{i \geq 8} k_{i} \leq 58 .
$$

Multiplication with 3/22 and rounding off yields Lemma 3. 


\section{Some preliminaries}

Assume $C \subset \mathbf{F}_{3}^{n}$ is a ternary code with covering radius one and $k=n-3$. We set

$$
\begin{gathered}
B_{i}=\left\{\sigma \in \mathbf{F}_{3}^{k}: n_{\sigma}=i\right\} \quad \text { for } \quad i=0,1, \\
B=B_{0} \cup B_{1}
\end{gathered}
$$

and

$$
\mathcal{K}_{i}=\{L \in \mathcal{L}:|L \cap B|=i\} \quad \text { for } \quad 0 \leq i \leq 3 .
$$

Let $c$ be a constant integer with $c \in\{5,6\}$. We set

$$
g^{*}(L)=g(L)-c \quad \text { for } \quad L \in \mathcal{L} .
$$

We find

$$
\begin{aligned}
\sum_{L \in \mathcal{L}} g^{*}(L) & =\sum_{0 \leq i \leq 3} \sum_{L \in \mathcal{K}_{i}} g^{*}(L) \\
& =\frac{1}{2} \sum_{1 \leq i \leq 3} i \sum_{L \in \mathcal{K}_{i}} g^{*}(L)+\frac{1}{2} \sum_{L \in \mathcal{K}_{1}} g^{*}(L)-\frac{1}{2} \sum_{L \in \mathcal{K}_{3}} g^{*}(L)+\sum_{L \in \mathcal{K}_{0}} g^{*}(L) \\
& =\frac{1}{2} \sum_{\sigma \in B} \sum_{L \in \mathcal{L}, \sigma \in L} g^{*}(L)+\frac{1}{2} \sum_{\sigma \in B} \sum_{L \in \mathcal{K}_{1}, \sigma \in L} g^{*}(L)-\frac{1}{2} \sum_{L \in \mathcal{K}_{3}} g^{*}(L)+\sum_{L \in \mathcal{K}_{0}} g^{*}(L)
\end{aligned}
$$

because in the sum $\sum_{\sigma \in B} \sum_{L \in \mathcal{L}, \sigma \in L} g^{*}(L)$ every $g^{*}(L)$ with $L \in \mathcal{L}$ and $|L \cap B|=i$ $(1 \leq i \leq 3)$ is counted exactly $i$ times. We now set

$$
B^{*}=\left\{\sigma \in B: \sum_{L \in \mathcal{L}, \sigma \in L} g^{*}(L)+\sum_{L \in \mathcal{K}_{1}, \sigma \in L} g^{*}(L)<0\right\} .
$$

By $c \in\{5,6\}$ we have $g^{*}(L) \leq-2$ for $L \in \mathcal{K}_{3}$ and $g^{*}(L) \geq 0$ for $L \in \mathcal{K}_{0}$. Thus from (10) and (11) now follows

$$
\sum_{L \in \mathcal{L}} g^{*}(L) \geq \frac{1}{2} \sum_{\sigma \in B^{*}}\left\{\sum_{L \in \mathcal{L}, \sigma \in L} g^{*}(L)+\sum_{L \in \mathcal{K}_{1}, \sigma \in L} g^{*}(L)\right\} .
$$

\section{$4 \quad$ Proof of $k_{3}(7) \geq 155$}

Assume $C \subset \mathbf{F}_{3}^{7}$ is a ternary code of covering radius one with $|C| \leq 155$. We set $k=4$ and choose $c=6$ in (9). In this section we show

$$
\sum_{L \in \mathcal{L}} g^{*}(L) \geq-28
$$


This suffices for a proof of the bound $k_{3}(7) \geq 155$. Assume (for the moment) $|C| \leq 154$. We have

$$
\begin{aligned}
4|C| & =4 \sum_{\sigma \in \mathbf{F}_{3}^{4}} n_{\sigma}=\sum_{\sigma \in \mathbf{F}_{3}^{4}} n_{\sigma} \sum_{L \in \mathcal{L}, \sigma \in L} 1=\sum_{L \in \mathcal{L}} g(L) \\
& =\sum_{L \in \mathcal{L}} g^{*}(L)+6 \sum_{L \in \mathcal{L}} 1 \geq-28+6 \cdot 108
\end{aligned}
$$

by (2), (3), (9) and (13) and thus $|C| \geq 155$, a contradiction. In the same way we get the bound $k_{3}(7) \geq 156$ if we are able to show that equality cannot hold in (13). This requires a more detailed analysis and is postponed to the next section. The reader interested in this bound should keep track about some consequences of assumed equality in (13) throughout this and the previous section. These consequences are listed in the beginning of section 5 .

We now show (13). From Lemma 1B and (2) we get

$$
\begin{array}{ll}
\sum_{L \in \mathcal{L}, \sigma \in L} g^{*}(L) \geq 0 & \text { for } \sigma \in B_{1}, \\
\sum_{L \in \mathcal{L}, \sigma \in L} g^{*}(L) \geq 3 & \text { for } \sigma \in B_{0} .
\end{array}
$$

Now assume $\sigma \in B^{*} \cap B_{1}$. If $L \in \mathcal{K}_{1}$ with $\sigma \in L$ and $g^{*}(L)<0$, then $L$ has value distribution $(1,2,2)$ and $g^{*}(L)=-1$. By (14) this can be satisfied for at most three of the four lines $L \in \mathcal{L}$ with $\sigma \in L$. Thus by (11) and (14) we may write

$$
\begin{gathered}
B^{*} \cap B_{1}=B_{1}^{*} \cup B_{2}^{*} \cup B_{3}^{*} \quad \text { with } \\
B_{i}^{*}=\left\{\sigma \in B^{*} \cap B_{1}: \sum_{L \in \mathcal{K}_{1}, \sigma \in L} g^{*}(L)=-i\right\} \quad \text { for } 1 \leq i \leq 3 .
\end{gathered}
$$

Moreover

if $\sigma \in B_{i}^{*}(1 \leq i \leq 3)$, then there exist at least $i$ lines $L \in \mathcal{K}_{1}$ with $\sigma \in L$ and value distribution $(1,2,2)$ (i.e. $g^{*}(L)=-1$ ).

If $\sigma \in B^{*} \cap B_{0}$ we have $g^{*}(L) \geq-2$ for $L \in \mathcal{K}_{1}$ with $\sigma \in L$. Like above by (15) at most three of the four lines $L \in \mathcal{L}$ with $\sigma \in L$ satisfy $g^{*}(L)<0$. Therefore

$$
\sum_{L \in \mathcal{K}_{1}, \sigma \in L} g^{*}(L) \geq \sum_{\substack{L \in \mathcal{K}_{1}, \sigma \in L \\ g^{*}(L)<0}} g^{*}(L) \geq-2 \sum_{\substack{L \in \mathcal{K}_{1}, \sigma \in L \\ g^{*}(L)<0}} 1 \geq-6 .
$$

This, together with (12), (14), (15) and (16) implies

$$
\sum_{L \in \mathcal{L}} g^{*}(L) \geq-\frac{1}{2}\left|B_{1}^{*}\right|-\left|B_{2}^{*}\right|-\frac{3}{2}\left|B_{3}^{*}\right|-\frac{3}{2}\left|B^{*} \cap B_{0}\right| .
$$


Lemma 4 a) If $\sigma \in B_{1}^{*}$, then there exists $L \in \mathcal{L}_{\geq 5}$ (see (4)) with $\sigma \in L$.

b) If $\sigma \in B_{2}^{*}$, then either there exists $L \in \mathcal{L}_{\geq 6}$ with $\sigma \in L$ or distinct $L_{1}, L_{2} \in \mathcal{L}_{5}$ with $\sigma \in L_{1} \cap L_{2}$.

c) If $\sigma \in B_{3}^{*}$, then there exists $L \in \mathcal{L}_{\geq 7}$ with $\sigma \in L$.

d) If $\sigma \in B^{*} \cap B_{0}$, then there exists $L \in \mathcal{L}_{\geq 8}$ with $\sigma \in L$.

Proof. a) If $\sigma \in B_{1}^{*}$, then by (14) and (16) there exists $L \in \mathcal{L} \backslash \mathcal{K}_{1}$ with $\sigma \in L$ and $g^{*}(L) \geq 1$, i.e. $g(L) \geq 7$. Suppose $L=\{\sigma, \rho, \tau\}$. By $\sigma \in B$ and $L \notin \mathcal{K}_{1}$ we have $|L \cap B| \geq 2$. Therefore we may assume that, say $\rho \in B$ and thus $n_{\sigma}+n_{\rho} \leq 2$. But by $g(L)=n_{\sigma}+n_{\rho}+n_{\tau} \geq 7$ we find $n_{\tau} \geq 5$, which means $L \in \mathcal{L}_{\geq 5}$ and a) follows.

b) If $\sigma \in B_{2}^{*}$, then by (14) and (16) there exists either $L \in \mathcal{L} \backslash \mathcal{K}_{1}$ with $\sigma \in L$ and $g^{*}(L) \geq 2$ or distinct $L_{1}, L_{2} \in \mathcal{L} \backslash \mathcal{K}_{1}$ with $\sigma \in L_{1} \cap L_{2}$ and $g^{*}\left(L_{1}\right)=g^{*}\left(L_{2}\right)=1$. In the first case like in a) we find $L \in \mathcal{L}_{\geq 6}$ and in the second case $L_{1}, L_{2} \in \mathcal{L}_{5}$ (if $L_{1}, L_{2} \notin \mathcal{L}_{\geq 6}$ ) and b) follows.

c) If $\sigma \in B_{3}^{*}$, then by (2), (14), (16) and (17) there exists a line $L \in \mathcal{L} \backslash \mathcal{K}_{1}$ with $\sigma \in L$ and $g^{*}(L) \geq 3$ and thus $L \in \mathcal{L}_{\geq 7}$ like in a).

d) If $\sigma \in B^{*} \cap B_{0}$, then by (11) and (15) we have $\sum_{L \in \mathcal{K}_{1}, \sigma \in L} g^{*}(L) \leq-4$ and $\sum_{L \in \mathcal{L} \backslash \mathcal{K}_{1}, \sigma \in L} g^{*}(L) \geq 7$. By $(2) \sum_{L \in \mathcal{L} \backslash \mathcal{K}_{1}, \sigma \in L} 1 \leq 3$ and thus there exists $L \in \mathcal{L} \backslash \mathcal{K}_{1}$ with $\sigma \in L$ and $g^{*}(L) \geq 3$. Like above $L \in \mathcal{L}_{\geq 8}$ follows by $n_{\sigma}=0$.

From Lemma 4 now follows

$$
\begin{aligned}
& \frac{1}{2}\left|B_{1}^{*}\right|+\left|B_{2}^{*}\right|+\frac{3}{2}\left|B_{3}^{*}\right|+\frac{3}{2}\left|B^{*} \cap B_{0}\right| \\
\leq & \frac{1}{2} \sum_{L \in \mathcal{L}_{\geq 5}}\left|B_{1}^{*} \cap L\right|+\sum_{L \in \mathcal{L}_{\geq 6}}\left|B_{2}^{*} \cap L\right|+\frac{1}{2} \sum_{L \in \mathcal{L}_{5}}\left|B_{2}^{*} \cap L\right| \\
& +\frac{3}{2} \sum_{L \in \mathcal{L}_{\geq 7}}\left|\left(B_{3}^{*} \cup\left(B^{*} \cap B_{0}\right)\right) \cap L\right| \\
= & \frac{1}{2} \sum_{L \in \mathcal{L}_{5}}\left|\left(B_{1}^{*} \cup B_{2}^{*}\right) \cap L\right|+\sum_{L \in \mathcal{L}_{6}}\left\{\frac{1}{2}\left|B_{1}^{*} \cap L\right|+\left|B_{2}^{*} \cap L\right|\right\} \\
& +\frac{3}{2} \sum_{L \in \mathcal{L}_{\geq 7}}\left\{\frac{1}{3}\left|B_{1}^{*} \cap L\right|+\frac{2}{3}\left|B_{2}^{*} \cap L\right|+\left|\left(B_{3}^{*} \cup\left(B^{*} \cap B_{0}\right)\right) \cap L\right|\right\} \\
\leq & \frac{1}{2} \sum_{L \in \mathcal{L}_{5}}\left|B^{*} \cap L\right|+\sum_{L \in \mathcal{L}_{6}}\left|B^{*} \cap L\right|+\frac{3}{2} \sum_{L \in \mathcal{L}_{\geq 7}}\left|B^{*} \cap L\right| \\
\leq & \left|\mathcal{L}_{5}\right|+2\left|\mathcal{L}_{6}\right|+3\left|\mathcal{L}_{\geq 7}\right| \quad \text { by }\left|B^{*} \cap L\right| \leq 2 \text { for } L \in \mathcal{L}_{\geq 5} \\
\leq & 4\left(k_{5}+2 k_{6}+3 \sum_{i \geq 7} k_{i}\right) \quad \text { by }(2) \\
\leq & 28 \quad \text { by Lemma } 3 \quad
\end{aligned}
$$

and (13) follows by (18). 


\section{Proof of Theorem 1}

Let $C \subset \mathbf{F}_{3}^{7}$ be a ternary code of covering radius one with $|C|=155$. Assume equality in (13). Then the following facts are necessarily satisfied (see the sections 3 and 4):

A) $g^{*}(L)=0$ whenever $L \in \mathcal{K}_{0}$.

B) $\quad \sigma \in B^{*} \cap B_{1}$ implies $\sum_{L \in \mathcal{L}, \sigma \in L} g^{*}(L)=0$.

C) $\quad L \in \mathcal{L}_{5}$ implies $\left|\left(B_{1}^{*} \cup B_{2}^{*}\right) \cap L\right|=2$.

D) $\quad L \in \mathcal{L}_{6}$ implies $\left|B_{2}^{*} \cap L\right|=2$.

E) $\quad L \in \mathcal{L}_{\geq 7}$ implies $\left|\left(B_{3}^{*} \cup\left(B^{*} \cap B_{0}\right)\right) \cap L\right|=2$.

F) $\quad k_{5}+2 k_{6}+3 \sum_{i \geq 7} k_{i}=7$.

We now use these facts to derive further properties of $C$ with the intention to get a contradiction.

G) $\quad k_{i}=0$ for $i \geq 8$.

Assume to the contrary that $\sum_{i \geq 8} k_{i}>0$ holds. From F) follows $\sum_{i \geq 8} k_{i} \leq 2$. If equality holds, then by (8) $k_{5}=k_{6}=\bar{k}_{7}=0$ and thus $k_{5}+2 k_{6}+3 \sum_{i \geq 7} k_{i}=6$ contradicting F). If $\sum_{i \geq 8} k_{i}=1$, then by (8) we have $8 k_{5}+15 k_{6}+22 k_{7} \leq 29$. Multiplication with $3 / 22$ and rounding off yields $k_{5}+2 k_{6}+3 k_{7} \leq 3$ and thus $k_{5}+2 k_{6}+3 \sum_{i \geq 7} k_{i} \leq 6$ again contradicting F).

H) $\quad B^{*} \cap B_{0}=\emptyset$.

This immediately follows from $\mathrm{G}$ ) and Lemma $4 \mathrm{~d}$ ).

I) $\quad 8 k_{5}+15 k_{6}+22 k_{7} \geq 52$.

Assume to the contrary $8 k_{5}+15 k_{6}+22 k_{7} \leq 51$. Multiplication with $3 / 22$ and rounding off would give $k_{5}+2 k_{6}+3 k_{7} \leq 6$ contradicting F) and $\mathrm{G}$ ).

J) If $\sigma, \rho \in \mathbf{F}_{3}^{4}$ with $n_{\rho} \geq 5$ and $d(\sigma, \rho)=1$, then $\sigma \in B^{*} \cap B_{1}$. Especially $n_{\sigma}=1$.

There exists $L \in \mathcal{L}$ with $\sigma, \rho \in L$. By $n_{\rho} \geq 5$ we have $L \in \mathcal{L}_{\geq 5}$. By C), D), E) and H) $\sigma \in B_{1}^{*} \cup B_{2}^{*} \cup B_{3}^{*}=B^{*} \cap B_{1}$ (see (16)).

K) If $\rho, \tau \in \mathbf{F}_{3}^{4}$ with $n_{\rho}=7$ and $d(\rho, \tau)=2$, then $n_{\tau}=2$.

Choose $\sigma \in \mathbf{F}_{3}^{4}$ with $d(\sigma, \rho)=1$ and $d(\sigma, \tau)=1$. By E) and $\left.\mathrm{H}\right)$ we have $\sigma \in B_{3}^{*}$. By (17) and $\mathrm{J})$ the four lines $L \in \mathcal{L}$ with $\sigma \in L$ have value distribution $(1,2,2),(1,2,2)$, $(1,2,2)$ and $(1,1,7)$ and $\mathrm{K})$ follows.

L) If $\sigma, \rho \in \mathbf{F}_{3}^{4}$ with $n_{\rho} \in\{5,6\}$ and $d(\sigma, \rho)=1$, then there exists $\tau \in \mathbf{F}_{3}^{4}$ with $d(\rho, \tau)=2, d(\sigma, \tau)=1$ and $n_{\tau} \geq 3$.

Assume $L \in \mathcal{L}$ with $\sigma, \rho \in L$. By J) and $n_{\rho} \in\{5,6\}$ we have $\sigma \in B_{1}$ and $g^{*}(L) \leq 2$. By (2) and (14) there exists a line $L^{\prime} \neq L$ with $\sigma \in L^{\prime}$ and $g^{*}\left(L^{\prime}\right) \geq 0$, i.e. $g\left(L^{\prime}\right) \geq 6$. By $n_{\sigma}=1$ there exists $\tau \in L^{\prime}$ with $n_{\tau} \geq 3$. We have $d(\sigma, \tau)=1$ by $\sigma, \tau \in L^{\prime}$ and $d(\rho, \tau)=2$ by $L^{\prime} \neq L$.

M) Whenever $\rho \in \mathbf{F}_{3}^{4}$ with $n_{\rho} \geq 5$, then $C \cap A_{\rho}$ 1-covers at most 25 from the 27 elements of $A_{\rho}$.

To see this, take any $L \in \mathcal{L}$ with $\rho \in L$. Assume $L=\left\{\rho, \sigma_{1}, \sigma_{2}\right\}$. By J) we have $\sigma_{1}, \sigma_{2} \in B^{*} \cap B_{1}$. For $i=1,2$ let $\mu_{i}=\left(\sigma_{i}, \kappa_{i}\right) \in C$ with $\kappa_{i} \in \mathbf{F}_{3}^{3}$ be the unique 
codeword with $h_{k}\left(\mu_{i}\right)=\sigma_{i}$. Now $\mu_{2}=\left(\sigma_{2}, \kappa_{2}\right)$ cannot 1-cover $\left(\sigma_{2}, \kappa_{1}\right)$ since this would imply that $\mu_{1}=\left(\sigma_{1}, \kappa_{1}\right)$ 1-covers $\left(\sigma_{1}, \kappa_{2}\right)$ and, following the proof of Lemma 1A (with $\sigma_{1}$ instead of $\left.\sigma\right)$, we see that $\mu_{2} \in C \cap A_{\sigma_{2}}$ then cannot contribute to the 1 -covering of $A_{\sigma_{1}}$ despite $d\left(\sigma_{1}, \sigma_{2}\right)=1$. This would result in an enlargement of the right-hand side of (6) to 28 , implying $\sum_{L \in \mathcal{L}, \sigma_{1} \in L} g(L) \geq 25$ (see Lemma 1B) and thus $\sum_{L \in \mathcal{L}, \sigma_{1} \in L} g^{*}(L) \geq 1$ contradicting B). Especially we have $\kappa_{1} \neq \kappa_{2}$. In the same way we see, that $\left(\rho, \kappa_{1}\right) \in A_{\rho}$ cannot be 1 -covered by $C \cap A_{\rho}$. By reasons of symmetry this also holds for $\left(\rho, \kappa_{2}\right) \in A_{\rho}$ and M) follows.

N) Either $k_{4}=k_{6}=0, k_{5}=1, k_{7}=2$ or $k_{4}=k_{5}=0, k_{6}=2, k_{7}=1$.

By M) we may apply Lemma 2 with $n=7, s=25$ and $|C|=155$. We get $k_{4}+10 k_{5}+$ $17 k_{6}+24 k_{7} \leq 58$. Subtracting the inequality from I) yields $k_{4}+2\left(k_{5}+k_{6}+k_{7}\right) \leq 6$. Especially $k_{5}+k_{6}+k_{7} \leq 3$. Subtracting this from $k_{5}+2 k_{6}+3 k_{7}=7$ (by F) and G)) yields $k_{6}+2 k_{7} \geq 4$. Now $k_{7}=0$ is not possible, since this would imply $k_{6} \geq 4$ contradicting $k_{5}+k_{6}+k_{7} \leq 3$. Also $k_{7}>2$ is not possible by $\mathrm{F}$ ). The case $k_{7}=2$ gives the first case and $k_{7}=1$ the second.

O) If $\rho, \tau \in \mathbf{F}_{3}^{4}$ with $n_{\rho}=6$ and $d(\rho, \tau)=2$, then $n_{\tau}=2$ or $n_{\tau}=3$.

Choose $\sigma \in \mathbf{F}_{3}^{4}$ with $d(\sigma, \rho)=1$ and $d(\sigma, \tau)=1$. Let $L^{\prime} \in \mathcal{L}$ be the line containing $\sigma$ and $\rho$. By J) we see, that $L^{\prime}$ has value distribution $(1,1,6)$ and $L^{\prime} \in \mathcal{L}_{6}$. Thus $\sigma \in B_{2}^{*}$ by D). By (17) the four lines $L \in \mathcal{L}$ with $\sigma \in L$ have value distribution $(1,2,2),(1,2,2)$, $\left(1, x_{1}, x_{2}\right)$ and $(1,1,6)$. Now B) implies $x_{2}+x_{3}=5$. By N) we have $k_{4}=k_{5}=0$ if $k_{6}>0$, thus $x_{1}, x_{2} \in\{2,3\}$ and $\left.\mathrm{O}\right)$ follows.

P) $\quad d\left(\rho_{1}, \rho_{2}\right)=4$ whenever $\rho_{1}, \rho_{2} \in \mathbf{F}_{3}^{4}$ with $5 \leq n_{\rho_{1}}, n_{\rho_{2}} \leq 7$ and $\rho_{1} \neq \rho_{2}$.

By N) either $n_{\rho_{1}} \geq 6$ or $n_{\rho_{2}} \geq 6$, say $n_{\rho_{1}} \geq 6$. J), K) and O) imply $d\left(\rho_{1}, \rho_{2}\right) \geq 3$. Now assume $d\left(\rho_{1}, \rho_{2}\right)=3$. Choose $\tau \in \mathbf{F}_{3}^{4}$ with $d\left(\rho_{1}, \tau\right)=2$ and $d\left(\rho_{2}, \tau\right)=1$. Now $d\left(\rho_{1}, \tau\right)=2$ implies $n_{\tau}>1$ by $\mathrm{K}$ ) and $\left.\mathrm{O}\right)$, whereas $d\left(\rho_{2}, \tau\right)=1$ implies $n_{\tau}=1$ by $\left.\mathrm{J}\right)$, a contradiction. Thus $d\left(\rho_{1}, \rho_{2}\right)=4$.

We now are in a position to derive a contradiction. Use N) to choose pairwise different $\rho_{1}, \rho_{2}, \rho_{3} \in \mathbf{F}_{3}^{4}$ with $5 \leq n_{\rho_{1}}, n_{\rho_{2}}, n_{\rho_{3}} \leq 7$. Assume $n_{\rho_{1}} \leq n_{\rho_{2}} \leq n_{\rho_{3}}$. By N) we have $n_{\rho_{1}} \leq 6, n_{\rho_{2}} \geq 6$ and $n_{\rho_{3}}=7$. By P) we may assume $\rho_{1}=(0000), \rho_{2}=(1111)$ and $\rho_{3}=(2222)$. Now consider $\sigma=(2000)$. By L) there exists $\tau \in \mathbf{F}_{3}^{4}$ with $d\left(\rho_{1}, \tau\right)=2$, $d(\sigma, \tau)=1$ and $n_{\tau} \geq 3$, say $\tau=(2100)$ (the case $\tau=(2200)$ is excluded by $\left.\mathrm{K}\right)$ ). Now consider the line $L=L(\tau, 3)=\{(2100),(2110),(2120)\}$. By K) and O) we have $n_{(2110)}, n_{(2120)} \geq 2$. Altogether we have $L \in \mathcal{K}_{0}$ and $g(L) \geq 7$, i.e. $g^{*}(L) \geq 1$ contradicting A). Thus equality cannot hold in (13). As we have seen in the previous paragraph, this implies Theorem 1. 


\section{Proof of Theorem 2}

Assume $C \subset \mathbf{F}_{3}^{8}$ is a ternary code of covering radius one with $|C|=401$. We follow section 3 with $k=5$ and choose $c=5$ in (9). By Lemma 1B (with $n=8$ ), (2) and (9) we have

$$
\begin{array}{ll}
\sum_{L \in \mathcal{L}, \sigma \in L} g^{*}(L) \geq 0 & \text { for } \sigma \in B_{1}, \\
\sum_{L \in \mathcal{L}, \sigma \in L} g^{*}(L) \geq 2 & \text { for } \sigma \in B_{0} .
\end{array}
$$

If $\sigma \in B_{1}$ we have $g^{*}(L) \geq 0$ for $L \in \mathcal{K}_{1}$ with $\sigma \in L$. This together with (11) and (19) implies

$$
B^{*} \subset B_{0} \text {. }
$$

Now assume $\sigma \in B^{*}$ and $L \in \mathcal{K}_{1}$ with $\sigma \in L$ and $g^{*}(L)<0$. Then $g^{*}(L)=-1$. By (20) and (21) at most four of the five lines $L \in \mathcal{L}$ with $\sigma \in L$ have this property. Therefore by (11), (20) and (21) we may write

$$
\begin{aligned}
& B^{*}=B_{3}^{*} \cup B_{4}^{*} \quad \text { with } \\
& B_{i}^{*}=\left\{\sigma \in B^{*}: \sum_{L \in \mathcal{K}_{1}, \sigma \in L} g^{*}(L)=-i\right\} \quad \text { for } \quad i \in\{3,4\} .
\end{aligned}
$$

Altogether now from (12), (20), (21) and (22) follows

$$
\sum_{L \in \mathcal{L}} g^{*}(L) \geq-\frac{1}{2}\left|B_{3}^{*}\right|-\left|B_{4}^{*}\right| .
$$

Lemma 5 a) If $\sigma \in B_{3}^{*}$, then there exists $L_{\sigma} \in \mathcal{L}$ with $\sigma \in L$, such that either $L_{\sigma} \in \mathcal{L}_{\geq 8}$ or $L_{\sigma} \in \mathcal{L}_{7}$ and $\left|B_{3}^{*} \cap L\right|=1$.

b) If $\sigma \in B_{4}^{*}$, then there exists $L_{\sigma} \in \mathcal{L}$ with $\sigma \in L$, such that either $L_{\sigma} \in \mathcal{L}_{\geq 11}$ or $L_{\sigma} \in \mathcal{L}_{10}$ and $\left|B_{4}^{*} \cap L\right|=1$.

Proof. a) If $\sigma \in B_{3}^{*}$, then $\sum_{L \in \mathcal{L} \backslash \mathcal{K}_{1}, \sigma \in L} g^{*}(L) \geq 5$ by (20), (21) and (22). By (22) and $g^{*}(L) \geq-1$ for $L \in \mathcal{K}_{1}$ at most two of the five lines $L \in \mathcal{L}$ with $\sigma \in L$ belong to $\mathcal{L} \backslash \mathcal{K}_{1}$. Therefore there exists $L_{\sigma} \in \mathcal{L} \backslash \mathcal{K}_{1}$ with $\sigma \in L_{\sigma}$ and $g^{*}\left(L_{\sigma}\right) \geq 3$, i.e. $g\left(L_{\sigma}\right) \geq 8$. By $L_{\sigma} \notin \mathcal{K}_{1}$ and $\sigma \in B$ we may assume $L_{\sigma}=\{\sigma, \mu, \lambda\}$ with $\mu \in B$, i.e. $n_{\mu} \leq 1$. By (21) we have $n_{\sigma}=0$. If $n_{\mu}=0$ then from $g\left(L_{\sigma}\right) \geq 8$ we find $n_{\lambda} \geq 8$, i.e. $L_{\sigma} \in \mathcal{L}_{\geq 8}$. If $n_{\mu}=1$ we find $\left|B_{3}^{*} \cap L_{\sigma}\right|=1$ and $L_{\sigma} \in \mathcal{L}_{7}$ (if $L \notin \mathcal{L}_{\geq 8}$ ). This completes the proof of a).

b) If $\sigma \in B_{4}^{*}$, then we see like in a), that there exists $L_{\sigma} \in \mathcal{L} \backslash \mathcal{K}_{1}$ with $\sigma \in L_{\sigma}$ and $g\left(L_{\sigma}\right) \geq 11$. The rest of the proof proceeds exactly like in a).

We set $\mathcal{L}^{\prime}=\left\{L_{\sigma} \in \mathcal{L}: \sigma \in B^{*}\right\}$. By Lemma $5 L \in \mathcal{L}_{7} \cap \mathcal{L}^{\prime}$ implies $\left|B_{3}^{*} \cap L\right| \leq 1$. Likewise we have $\left|B_{4}^{*} \cap L\right| \leq 1$ for $L \in \mathcal{L}_{10} \cap \mathcal{L}^{\prime}$, and thus $\frac{1}{2}\left|B_{3}^{*} \cap L\right|+\left|B_{4}^{*} \cap L\right| \leq \frac{3}{2}$ for 
$L \in \mathcal{L}_{10} \cap \mathcal{L}^{\prime}$. Using these remarks from Lemma 5 now follows

$$
\begin{aligned}
\frac{1}{2}\left|B_{3}^{*}\right|+\left|B_{4}^{*}\right| \leq & \frac{1}{2} \sum_{L \in \mathcal{L}_{\geq 7} \cap \mathcal{L}^{\prime}}\left|B_{3}^{*} \cap L\right|+\sum_{L \in \mathcal{L}_{\geq 10} \cap \mathcal{L}^{\prime}}\left|B_{4}^{*} \cap L\right| \\
= & \frac{1}{2} \sum_{L \in \mathcal{L}_{7} \cap \mathcal{L}^{\prime}}\left|B_{3}^{*} \cap L\right|+\frac{1}{2} \sum_{8 \leq i \leq 9} \sum_{L \in \mathcal{L}_{i} \cap \mathcal{L}^{\prime}}\left|B_{3}^{*} \cap L\right| \\
& +\sum_{L \in \mathcal{L}_{10} \cap \mathcal{L}^{\prime}}\left(\frac{1}{2}\left|B_{3}^{*} \cap L\right|+\left|B_{4}^{*} \cap L\right|\right)+\sum_{L \in \mathcal{L}_{\geq 11} \cap \mathcal{L}^{\prime}}\left(\frac{1}{2}\left|B_{3}^{*} \cap L\right|+\left|B_{4}^{*} \cap L\right|\right) \\
\leq & \frac{1}{2}\left|\mathcal{L}_{7} \cap \mathcal{L}^{\prime}\right|+\sum_{8 \leq i \leq 9}\left|\mathcal{L}_{i} \cap \mathcal{L}^{\prime}\right|+\frac{3}{2}\left|\mathcal{L}_{10} \cap \mathcal{L}^{\prime}\right|+2 \sum_{i \geq 11}\left|\mathcal{L}_{i} \cap \mathcal{L}^{\prime}\right| \\
& \left(\text { by }\left|B^{*} \cap L\right| \leq 2 \quad \text { for } \quad L \in \mathcal{L}_{\geq 7}\right) \\
\leq & \frac{1}{2}\left|\mathcal{L}_{7}\right|+\left|\mathcal{L}_{8}\right|+\left|\mathcal{L}_{9}\right|+\frac{3}{2}\left|\mathcal{L}_{10}\right|+2 \sum_{i \geq 11}\left|\mathcal{L}_{11}\right| \\
\leq & \frac{5}{2} k_{7}+5 k_{8}+5 k_{9}+\frac{15}{2} k_{10}+10 \sum_{i \geq 11} k_{i} \quad \text { by }(2) \\
\leq & \frac{1}{5}\left(22 k_{7}+29 k_{8}+36 k_{9}+43 k_{10}+50 \sum_{i \geq 11} k_{i}\right) \\
< & 20 \quad \text { by Lemma } 2(\text { with } n=8, s=27,|C|=401) .
\end{aligned}
$$

We now complete the proof of Theorem 2. We have

$$
\begin{aligned}
5|C| & =5 \sum_{\sigma \in \mathbf{F}_{3}^{5}} n_{\sigma}=\sum_{\sigma \in \mathbf{F}_{3}^{5}} n_{\sigma} \sum_{L \in \mathcal{L}, \sigma \in L} 1=\sum_{L \in \mathcal{L}} g(L) \\
& =\sum_{L \in \mathcal{L}} g^{*}(L)+5 \sum_{L \in \mathcal{L}} 1>-20+5 \cdot 405
\end{aligned}
$$

by (2), (3), (9), (23) and (24) and thus $|C|>401$, a contradiction. This completes the proof of Theorem 2.

\section{Acknowledgement}

I wish like to thank Michael Botzet (Hamburg), who brought me to the problem. I am also grateful to the anonymous referee for helpful comments.

\section{References}

[1] G. Cohen, I.S. Honkala, S. Litsyn, A. Lobstein, Covering codes, North Holland Mathematical Library, vol 54, 1997, Elsevier. 
[2] W. HAAs, Lower bounds for q-ary codes of covering radius one, Discr. Mathematics 219 (2000), 97-106.

[3] W. HAAs, Binary and ternary codes with covering radius one: Some new lower bounds, Discr. Mathematics 256 (2002), 161-178.

[4] L. HABSIEGER, Lower bounds for q-ary coverings by spheres of radius one, J. Comb. Theory Ser. A 67 (1994), 199-222.

[5] L. Habsieger, A new lower bound for the football pool problem for 7 matches, J. de Th. des Nombres de Bordeaux 8 (1996), 481-484.

[6] L. Habsieger, A. Plagne, New lower bounds for covering codes, Discr. Mathematics 222 (2000), 125-149.

[7] G. KÉRI, Tables for Covering Codes, http://www.sztaki.hu/ keri/codes/. 